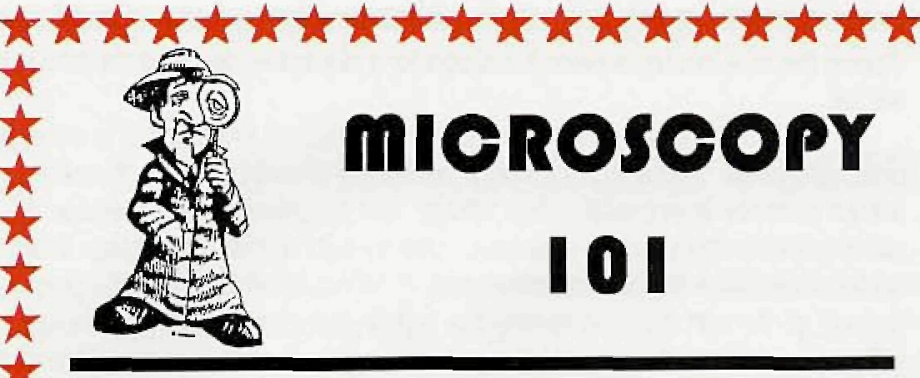

We continue to appreciate contributions to this new publication feature. in addition to items relating to electron microscopy, we hope to publish more material on other microscopy techniques. To do this, and hopefully make the feature of increasing interest and value, we need your assistance.

Contributions may be sent to Philip Oshel as follows:

eMail: oshel@ux1.cso.uiuc.edu

Snail Mail: Station A

PO Box 5037

Champaign IL 61825-5037

\section{EPON 812 \\ Spurr's Resin Mixture Formula:}

Prepare resin mixture the same day that it is to be used. Since infiltration takes more than one day, the stored resin mixture will begin to thicken by the second day (due to the presence of catalysts). Thus, fresh resin mixture should be prepared, and it is wasteful to prepare more than needed at any one time. Adjust volume according to the values given below. Une gram equals approximately $1.2 \mathrm{~mL}$.

Prepare resin mixture by weight using a top-loading balance that weighs accurately to at least two decimal places. Mix resins in plastic disposable beakers and add resins with strong pipet head to the beakers using Pasteur pipets. When mixing resins, do not worry about air bubbles. This will not be a factor in the infiltration steps. However, the final pure resin mixture for embedding should be free of air bubbles.
A. Epon 812 (or substitute such as Epox 812)
$5.0 \mathrm{gm}$.
DDSA
$2.8 \mathrm{gm}$.
NMA
$2.0 \mathrm{gm}$.
B. ERL (From Spurr Kit)
$2.2 \mathrm{gm}$.
DER " " "
$1.4 \mathrm{gm}$.
NSA "
$6.2 \mathrm{gm}$.
C. DMP-30
$0.2 \mathrm{gm}$.
DMAE
$0.2 \mathrm{gm}$.

Always use separate Pasteur pipets for each resin ingredient, and then dispose of them. Thoroughly mix ingredients in (A) above, together in a plastic beaker. Mix ingredients in $(B)$ above, separately in another plastic beaker. Add ingredients from beaker $(B)$ to those in beaker $(A)$ and mix thoroughly. (Mixing can be done by using 2-3 wooden applicator sticks to completely stir the medium until no swirl marks can be observed.) Add the ingredients listed under (C) directly to the combined mixture in beaker (A). Stir in completely the DMP-30 first; then thoroughly mix in the DMAE. Tightly cover the beaker with Parafilm ${ }^{T M}$ when not used. Store away from sunlight in desiccator. Storage time is limited to 24-30 hours at room temperature since it will become too thick to be effectively used. Always use resins which are not contaminated with moisture or organic residues.

When bottles are stoppered after pipetting needed amounts, clean the neck of each bottle with small amount of acetone on a cloth or Kimwipes gently inject a moderate amount of Freon, $\mathrm{CO}_{2}$ or other dy inert gas (from a duster can) into the bottle, and replace the cap without twisting too hard. Cover cap with tight strips of Parafil $\mathrm{m}^{\mathrm{TM}}$. Store in a cool (but not cold), dark location until next use. Most resin components last for several months to several years if properly stored at moderate temperatures and in an uncontaminated state.

After sectioning and staining, please remember that the best results are obtained when specimens are observed and micrographed in the transmission electron microscope as soon as possible.

Richard F.E. Crang, University of Illinois

Gold Toning for Silver Enhanced Immunogold Reacted Tissue:

Based on the method of Arai, R., M. Geffard and A. Calas, 1992. Intensification of labelings of the immunogold silver staining method by gold toning. Brain Research Bulletin 28:342-345.

Labeling tissue by the pre-embedding immunogold method and then silver enhancing the gold particles, gives beautiful staining ideal for both light microscopy and electron microscopy. However, after embedding the reacted tissue normally for TEM and cutting sections, it is common to find that most of the silver label has mysteriously disappeared. The reason is that the osmium tetroxide used during the embedding procedure oxidizes the silver which then becomes soluble. Fortunately, only the subsequent uranyl acetate step seems to actually wash the silver salt out of the tissue - it does not matter whether or not the uranyl acetate is dissolved in water or alcohol. So, if embedding tissue without uranyl acetate en bloc staining gives usable sections, the problem of the disappearing silver will be solved.

An alternative approach is to gold tone the tissue before embedding. Gold toning will add considerable contrast to the tissue, so that subsequent section staining can be reduced

Gold toning also makes the tissue rather "grainy", which shows up at higher magnifications, so keep reaction times to a minimum. The times quoted below have been found suitable for retinal tissue, which is around $300 \mu \mathrm{m}$ thick, and for "average" sized silver grains. If the gold toned silver particles appear to have a hollow center, then lengthen the reaction times. Note: this recipe assumes that your tissue has already been immunogold-reacted and silver enhanced!

1. Silver enhance immunogold reacted tissue and wash as usual for your tissue.

2. Fix in glutaraldehyde. Tissue can be stored indefinitely in cacodylate buffer after this step.

3. Rinse in water for about 30 minutes.

4. Incubate in $0.05 \%$ gold chloride 30 minutes at $4^{\circ} \mathrm{C}$.

5. Rinse in water 10 minutes

6. Incubate in $0.05 \%$ oxalic acid 5 minutes at room temperature

7. Rinse in water 10 minutes.

8. Incubate in freshly prepared $1 \%$ sodium thiosulphate for 1 hour.

9. Rinse in water for 10 minutes and embed normally for TEM

Diana van Driel, Sydney University (Australia)

\section{A Method for Making White Borders Around Black Text in Adobe PhotoShop:}

Dry transfer letters had one big advantage over computers for labeling micrographs: they were available as white-outlined black letters, and so could be seen against any background. We have learned how to do this in Photoshop. The following method assumes you are using version 4 on a Macintosh, although it Is possible in version 3 with minor modifications, and on a Wintel computer with slightly different keystrokes

1) With black as the foreground color, write the text (draw the arrow, etc.) 
Photoshop 4 creates a new layer each time text is written. For drawing an arrow, create a new layer first.

2) For the layer of interest, deselect the "preserve transparency" feature in the layers palette.

3) Make white your foreground color.

4) Holding down the "command" button ("ctrl" an a Wintel), click on the layer of interest in the layers palette. This selects the non-transparent areas of the layer (e.g., your text or feature).

5) Under the Edit menu, select "stroke". This will put a line of the foreground color, at a selected width, on the inside, outside, or center of the dashed "select" line in the image. A five pixel width for a 300 dpi image and "outside" are typical. 6) Select OK; this should give the text or feature a white border. This can be seen more easily (without deselecting the feature) by using the "Hide Edges" command (command-H for Macintosh). This removes the dashed 'select" line (though the feature is still selected), and allows a clean look at the work for proofing.

John Vetrano will welcome questions on this procedure and may be contacted at email address: js_vetrano@pnl.gov

John Vetrano, Pacific Northwest Laboratory

\section{Healing Cracks in Glycolmethacrylate Embedded Sections:}

Soak the slide with attached sections in a coplin jar with acetone for 10 minutes or so (I have gone up to 30 or 40 minutes), rinse with water, and then apply your stain while the sections are still wet.

Generally the cracks seem to disappear, or are at least minimized. and it no longer interferes with the microstructure of whatever l'm looking at.

I typically use this for sections of between 2 and $5 \mu \mathrm{m}$ sections for light microscopy.

I haven't figured out where the cracks come from. Initially I thought It was age, (old sections) but have also had problems with freshly sectioned material. (including freshly embedded and polymerized)

\section{S. Shea Miller, Agriculture \& Agri-Food Canada}

\section{Archival Storage of Polaroid Films}

The materials listed below contain a minimum of potentially harmful substances. Therefore, they are regarded as being among the more suitable for the storage of photographs.

+ Storage boxes made of acid-free paper or card - Cellulose acetate

+ Polyethylene

+ Polyester sheeting and sleevesas

+ Aluminum

+ Stainless Steel

+ Materials coated with baked enamel

+ Glass

+ Porcelain

+ Acrylic plastics

The following products should be avoided:

- Paper with a high content of sulfur compounds (such as some black album papers)

+ Brown kraft paper (contains lignin and metallic sulfates)

+ Unstable plastics (such as polyvinyl chloride and polystyrene)

+ Ordinary cardboard

+ Some uncoated and unaged wood, especially those containing glue (plywood or pressboard)

+ Wood recently varnished or painted

+ Glassine envelopes

+ Inks from pens or rubber stamps

+ Rubber bands(may contain sulfur)

+ Insecticides and fungicides

Philip J. Cravotta, Polaroid Technical Assistance

\section{Mounting Small Specimens for Making an Animated Sequence in the SEM:}

I'm doing an animated sequence from the SEM, in stereo, on a very small spider (a couple of thousand frames). The mounting technique is critical for doing this.

\section{Tip \#1: BSE detection and Carbon}

I'm shooting with a Robinson BSE detector. It gives a more "real" illumination than SE and has the benefit of not "seeing" carbon. The specimen is normally mounted on top of a minuten (entomological) pin which is clamped in a $12 \mathrm{~mm}$ "vice-stub" and tilted at 90 degrees in the $\mathrm{SEM}$. On my Cambridge $\mathrm{S} 120$, the top of the stage mechanism is now out of view but you still see the bottom plate below. I've covered that with a piece of aluminum foil which has been painted with thinned-out carbon dag, making it a perfectly black, "studio-shot" background when using BSE.

Tip \#2: No unsightly props

Further, instead of mounting the specimen on the usual minuten pin, I've fixed it to the end of the lead from a clutch pencil (draughtsman's pencil), so the pin is also virtually invisible under BSE.

Doesn't the pencil lead get sputter-coated too? I've taken a mediumbore tip from a syringe, held it in an alligator clip, and fed the pencil lead down into it leaving just the specimen exposed for coating.

Tip \#3: Accuracy in mounting

Mounting very small specimens on a pin-tip is difficult at the best of times. As the animation sequence is to be in stereo, the specimen must be at exactly the right angles to the pin and we don't want to see any excessive blobs of carbon dag. You'll need a small bench vice, micrometer screw gauge and double sided tape (DST). Lay your stereomicroscope down on its front with the head reversed so that the eyepieces point up and the light path is (roughly) horizontal. Clamp the side of the screw gauge into the side of the vice and place it in front of the stereoscope. Secure your specimen to a foam block with crossed minuten pins and secure the block in position to the opposing pole of the screw gauge with DST. Fix the mounting pin to the mobile shaft of the screw gauge with DST. Watching it all through the stereoscope, do a dummy run to ensure that everything lines up. Screw the pin up, apply a minute amount of dag to the pin, just enough to form a meniscus on the squared-off end of the pin (rather than a blob) and screw it down to the specimen. Leave to dry.

\section{Tip \#4: Centre of rotation}

Because a sequence may include rotating the animal 360 degrees, the pin has to be at the centre of rotation of the stage. However, in my 12 $\mathrm{mm}$ "vice-stub", the vice is offset. Instead, I'm using a pop-rivet. Push the nail out and use the rivet and its collar; the rivet has the same diameter as the shaft on a normal $12 \mathrm{~mm}$ grooved stub. Put the pin down the bore and dag it into a central position.

I hope you find these tips helpful and it you're in $\mathrm{Oz}$ around September, drop into the Australian Museum, Sydney, to see the stereo animation sequence of the spider (unfortunately, I'll be gone by then).

\section{Geoff Avern, Australian Museum, Sydney}

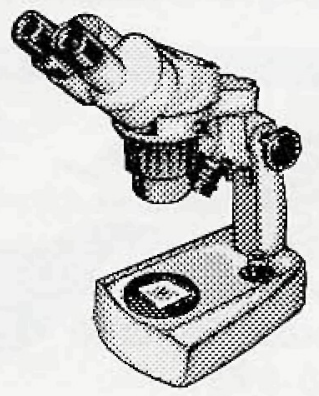

